Congreso Internacional de Gestión Educativa, 06 y 07 de noviembre de 2020.

Pontificia Universidad Católica del Perú

\title{
Gestión digital en la UGEL Puno
}

David Gregorio Cornejo Mamani

Director de la UGEL Puno

gredavicom7@hotmail.com

https://orcid.org/0000-0002-3231-0236

\author{
Choque Copari Rene \\ Jefe de AGP de la UGEL Puno \\ renecho21@yahoo.es \\ https://orcid.org/0000-0001-8711-1042
}

Resumen: La UGEL Puno, para mejorar el servicio educativo orientado a 52,553 estudiantes de 512 instituciones educativas y 341 programas no escolarizados de educación inicial, ha implementado el uso de la plataforma virtual: Sistema Integrado para Instituciones Educativas (SIIE) para coordinar la gestión con 853 Directores y 3,490 docentes de educación básica y técnico productiva, mediante la Resolución Directoral $\mathrm{N}^{\circ} 2469$ del 26 de abril 2019. Teniendo como base normas de convivencia andina.

Se fortalece el uso del SIIE en el marco de la emergencia sanitaria por el covid 19, desarrollando capacidades digitales en los directores y docentes de las instituciones educativas con asistencias técnicas como actividades virtuales denominado TECNO PUNO I - II, mejorando la utilización de herramientas y entornos virtuales para la optimizar la estrategia aprendo en casa, dispuesta por el MINEDU.

Palabras clave: Plataforma virtual; servicio educativo; emergencia sanitaria; Tecno Puno, estrategia aprendo en casa.

\section{Digital management in the UGEL Puno}

Abstract: UGEL Puno, to improve the educational service to 52,553 students in 512 educational institutions and 341 out-of-school programs of initial education, through RD No. 2469 of April 26, 2019 has implemented the use of the virtual platform: Integrated System for Educational Institutions (SIIE) to coordinate management with 853 Directors and 3,490 teachers of basic and technical productive education, based on Andean coexistence rules.

The use of SIIE is strengthened in the framework of the health emergency caused by Covid-19, developing digital abilities in directors and teachers of educational institutions with technical assistance such as virtual events called TECNO PUNO I - II, improving the use of tools and virtual environments to optimize the learning strategy " I learn at home" arranged by MINEDU.

Keywords: Virtual platform; educational service; health emergency; Techno Puno, learning strategy at home.

\section{Introducción:}

La Unidad de Gestión Educativa Local de Puno, es una instancia de ejecución descentralizada del Gobierno Regional con autonomía en el ámbito de su competencia y su jurisdicción territorial es la provincia de Puno. Dentro de sus funciones está la de contribuir a la formulación de políticas educativas para el espacio regional y nacional, 
Congreso Internacional de Gestión Educativa, 06 y 07 de noviembre de 2020.

Pontificia Universidad Católica del Perú

así como diseñar, ejecutar y evaluar el Proyecto Educativo Local de su jurisdicción en concordancia con los Proyectos Educativos Regionales y Nacionales.

La UGEL Puno, es la responsable de liderar la educación en la Provincia con una visión holística de cambio y desarrollo local, en esa línea el Proyecto Educativo Local Concertado 2020-2032, dinamiza las políticas educativas locales de la Provincia, articulando sus lineamiento al proyecto educativo regional y nacional.

\section{Ubicación geográfica}

Puno se ubica en la zona sur oriental del Perú, entre las siguientes coordenadas:

\begin{tabular}{|l|l|l|l|l|}
\hline ORIENTACIÓN & NORTE & \multicolumn{1}{l}{ ESTE } & SUR & OESTE \\
\hline Latitud sur & $13^{\circ} 00^{\prime} 00^{\prime \prime}$ & $16^{\circ} 19^{\prime} 00^{\prime \prime}$ & $17^{\circ} 17^{\prime} 30^{\prime \prime}$ & $14^{\circ} 42^{\prime} 47^{\prime \prime}$ \\
\hline Latitud oeste & $68^{\circ} 58^{\prime} 46^{\prime \prime}$ & $68^{\circ} 48^{\prime} 46^{\prime \prime}$ & $69^{\circ} 43^{\prime} 48^{\prime \prime}$ & $71^{\circ} 06^{\prime} 46^{\prime \prime}$ \\
\hline
\end{tabular}

Fuente: GRP. Año 2015.

En el centro meridional se ubica la provincia de Puno, con un área de 6,492.60 km2, dentro del denominado ecosistema altiplánico entre los ramales Occidental y Oriental de la cordillera de los andes, donde se distingue un área de influencia del Lago Titicaca constituido en $60 \%$ por pampas, llanuras o praderas y en $40 \%$ por pendientes y quebradas. Su formación ecológica predominante es el bosque húmedo montano (pajonal andino).

La zona circunlacustre alrededor del Lago Titicaca presenta condiciones favorables para el agro en terrenos de poca pendiente; el área de pradera tiene grandes extensiones de pastos que incentivan la actividad pecuaria.

\section{Limites:}

- Norte: provincia de San Román, Huancané y parte del Lago Titicaca.

- Sur: Provincia de el Collao y Dpto. Moquegua

- Este: Provincia de el Collao y el Lago Titicaca

- Oeste: Dpto. de Moquegua y Provincia de San Román

\section{Altitud y clima:}

La provincia de Puno, está ubicado a orillas del Lago Titicaca, el lago navegable más alto del mundo a 3827 m.s.n.m. Se encuentra en la región de la sierra a los $15^{\circ} 50^{\prime} 26^{\prime \prime}$ de latitud sur, $70^{\circ} 01^{\prime} 28^{\prime}$ ' de longitud Oeste del meridiano de Greenwich; ocupa una extensión de $460.63 \mathrm{~km} 2$. El clima se describe como lluvioso, frio y seco.

\section{Extensión territorial}

La provincia de Puno tiene una superficie aproximada de $6,492 \mathrm{~km} 2$, distribuidos entre distritos, comunidades campesinas, centros poblados y parcialidades. Está conformado 
Congreso Internacional de Gestión Educativa, 06 y 07 de noviembre de 2020.

Pontificia Universidad Católica del Perú

por 15 distritos: Puno, Acora, Amantani, Atuncolla, Capachica, Chucuito, Coata, Huata, Mañazo, Paucarcolla, Pichacani, Plateria, San Antonio, Tiquillaca y Vilque.

\section{La emergencia sanitaria}

Como en diferentes contextos, la repentina propagación del coronavirus y declaratoria de emergencia sanitaria, obligó al Ministerio de Educación emitir normas para la implementación de la estrategia "Aprendo en Casa" con la finalidad de garantizar la continuidad del servicio educativo en el año escolar 2020; sin embargo, la diversidad socio cultural, geográfica, social y económica del Perú e incluso la particularidad de las regiones exigían un tratamiento y adecuación de la estrategia y su aplicación en la práctica pedagógica, siendo una de los aspectos más importantes la utilización de herramientas virtuales por los docentes, padres de familia y estudiantes, sin considerar el equipamiento y conectividad mínima.

\section{Referentes Teóricos:}

\section{Enfoque del buen vivir:}

El Proyecto Educativo Regional de Puno (2017-2025) asume a la educación, como un proceso de construcción y formación integral del futuro ciudadano. Un proceso que permita el impulso de las potencialidades de los estudiantes para que contribuyan con el desarrollo de su entorno, pero sin perder la esencia del "buen vivir". Buen vivir que concibe al desarrollo, no como una simple adquisición de bienes y fomento del consumismo; sino, un buen vivir como proceso que permita la atención a las necesidades y al logro del bienestar del ser humano en armonía y respeto a la Pachamama y a su comunidad. Buen vivir que conciba el fortalecimiento de los lazos de la familia a partir de la práctica de valores andinos que se contrapongan a las nuevas tendencias extrañas, bajo el pretexto de la modernidad, fomentan estilos de vida consumista, de despilfarro, sin ninguna consideración por el cuidado de su entorno ambiental y social.

La educación que queremos se relaciona con el tipo de ciudadanos que aspiramos lograr; lo cual requiere una profunda reflexión como sociedad, acerca de las fortalezas que poseemos y que deseamos potencializar. Somos una Región admirada por la inmensa riqueza cultural, natural, productiva y espiritual, definida por la simbiosis hombre-naturaleza, biodiversidad natural, folklore, costumbres, danzas, gastronomía, historia; riquezas que permiten al puneño ser auténtico, sentirse orgulloso e identificado con su cultura y no necesita copiar algún patrón cultural extranjero.

Se concibe entonces al desarrollo como una construcción social a partir de un enfoque de desarrollo humano sostenible, no es posible entender el desarrollo si este no es humano, sino se refiere al mejor estar de la humanidad en la sociedad (Rendon, $2007 \mathrm{p}$. 1), sin que ello signifique el desentendimiento de la responsabilidad con el medio ambiente. Así, se aspira a la consecución de una sociedad justa, inclusiva, responsable con su entorno, con respeto hacia sus ciudadanos y a la Pachamama; una sociedad donde prime la mayor valoración del más querer y no la del más tener. Se concibe a la educación como un derecho más que como un servicio. En ese sentido, se concentran los esfuerzos en garantizar que todos tengan acceso y posibilidad de atención educativa de calidad. Una atención que esté acorde con los vertiginosos cambios científico- 
Congreso Internacional de Gestión Educativa, 06 y 07 de noviembre de 2020.

Pontificia Universidad Católica del Perú

tecnológicos que viene provocando el presente periodo de revolución del conocimiento en el que nos encontramos.

Puno, potencializa la producción agrícola, pecuaria, pesquera, artesanal, turística, minera, textil e industrial. Progreso meritorio, considerando las difíciles características geográficas de la Región, la misma que forjó un ciudadano puneño sin temor al esfuerzo y con gran dedicación al trabajo, sobre todo, con gran perseverancia por la superación de sí mismo y de los suyos. Por esa autenticidad cultural, espiritual y conductual, el puneño emerge como un ser humano ético, respetuoso de las normas morales, educado, preocupado por su comunidad, solidario, moral, propositivo, esforzado por el trabajo, perseverante, auténtico, identificado con los patrones positivos de su cultura, con amor por el arte y la belleza, reacio ante las injusticias, investigador e innovador; características con las que aspira lograr un buen vivir en armonía con la cosmovisión andina.

En este contexto, las tecnologías de la información y comunicación (TIC) se han convertido en una poderosa herramienta didáctica que promueven la colaboración entre los estudiantes, centrarse en sus aprendizajes y mejoran la motivación y el interés por el estudio. Asimismo, generan la integración y estimulan el desarrollo de ciertas habilidades intelectuales tales como el razonamiento, la resolución de problemas, la creatividad y la capacidad de aprender a aprender.

\section{Enfoques complementarios}

Enfoque de desarrollo humano. El desarrollo humano, según el PNUD (1990), “...es el proceso mediante el cual se amplían las oportunidades de las personas, para que disfruten de una vida larga y saludable, accedan a los conocimientos y recursos necesarios para llevar una vida digna...". En esa línea, Sen (1998) señala: "El enfoque de desarrollo humano es un gran avance en el difícil ejercicio de comprender los logros y las carencias de la vida humana, de reconocer la importancia de la reflexión y el diálogo, para alcanzar la justicia y equidad en el mundo".

Enfoque de desarrollo sostenible. Este enfoque anhela el desarrollo de los pueblos, considerando como fundamental los recursos naturales; en esa visión, la Comisión Brundland, en el documento "Nuestro futuro común" (1987) subraya, “...es un desarrollo que satisface las necesidades de la generación presente, sin comprometer la capacidad de las generaciones futuras para satisfacer sus propias necesidades". Con esta frase, se pretende promover el uso responsable de los recursos naturales frente a la explotación y destrucción de los ecosistemas que pone en peligro a la especie humana y a todo ser vivo en la Tierra.

Enfoque de educación basada en derechos humanos. No solo el derecho a la educación, también el acceso y su permanencia en igualdad de oportunidades con inclusión sin discriminación respetando sus entornos de aprendizaje, su identidad, la libre expresión y el respeto de su integridad física y moral. 
Enfoque territorial. El "territorio" no es solo un espacio físico-natural, sino un espacio socialmente construido; un ámbito habitado por comunidades y grupos humanos que comparten lazos históricos, simbólicos, culturales y económicos. Cuanto más "territorializada" sea la gestión educativa, se garantiza una mayor atención a cada contexto geográfico, social, cultural y lingüístico de los niños y las familias. Las potencialidades del desarrollo con enfoque territorial a nivel local; generan sinergias que pueden fortalecer a la escuela para la atención integral del infante con una mirada holística. (CNE, 2013).

Enfoque social comunitario. Con este enfoque, se revalora y se afirma la condición social del ser humano comunitario y espiritual que se desarrolla a partir de la relacionalidad con y entre los seres de la Pachamama. El PCR (2009) señala: "Los padres y los sabios de la comunidad deben participar de manera protagónica en la promoción de aprendizajes pertinentes y de calidad; estos sujetos de la educación, interactúan en el contexto social y cultural, según el grado de influencia y de acuerdo a su rol asignado a cada cual por la comunidad".

\section{Principios}

Equidad de género PCR (2009): "La equidad de género visto desde la complementariedad y relacionalidad entre el hombre y la mujer". En el mundo andino se concibe a la pareja en matrimonio como un principio perenne y fundamental de nuestra sociedad, por ello, la dualidad complementaria forma parte de la institucionalidad andina. El trato entre el hombre y la mujer es igualitario o equivalente, al que todos tienen derecho según el contexto.

Inclusión educativa: Todos los niños tienen el derecho fundamental, el acceso a la educación, el que no puede ser vulnerado bajo ningún argumento político, social, cultural o jurídico. En esa medida, el Estado garantiza a los infantes iguales oportunidades de acceso, permanencia y trato en un sistema educativo de calidad para contribuir a eliminar la pobreza, la exclusión y las desigualdades sociales.

Democracia participativa: Las personas son los actores principales para alcanzar el desarrollo y deben participar activamente de este proceso por ser beneficiarios del derecho al desarrollo. Según Pacheco (2006), todos los miembros de una comunidad tienen reglas y normas de comportamiento establecidas que les permite participar y relacionarse con los demás. Todos y todo pertenece a una sola familia bajo un solo techo.

Desarrollo sostenible: Vivir bien, es saber vivir en armonía y equilibrio con los ciclos de la madre tierra; asimismo implica, primero, saber vivir y luego convivir con la Pachamama. El desarrollo sostenible apunta a pensar en el futuro de las generaciones que vienen, garantizándoles los recursos naturales para su sobrevivencia, mediante el crecimiento ambiental, social, cultural y económico de manera sostenible. 
Congreso Internacional de Gestión Educativa, 06 y 07 de noviembre de 2020.

Pontificia Universidad Católica del Perú

Educación intercultural. Asume la diversidad cultural, étnica y lingüística como una posibilidad de riqueza, aceptando y respetando las diferencias para una convivencia armónica y de intercambio cultural a nivel local, nacional e internacional. La interculturalidad es un proceso de comunicación e interacción entre personas y grupos culturalmente diferenciados donde no se permite que un grupo esté por encima del otro, favoreciendo en todo momento el diálogo horizontal y la convivencia.

Ética y moral: Ser responsable es no transgredir las conductas y comportamientos establecidos por la familia y la comunidad. En esta línea es fundamental conservar aún las practicas simbólicas y materiales que buscan una relación equilibrada entre las personas y la naturaleza de la cultura ancestral, como el ayni, la minka y la mita, los que deben ponerse en práctica con el fin de respetar la vida, la libertad y la identidad.

Educación productiva: La región Puno, posee una gran riqueza natural y diversidad ecológica; entonces, la educación debe estar orientada a la adquisición y desarrollo de competencias laborales y empresariales para lograr su desarrollo ambiental, cultural, social y económico. Es necesario recuperar y revalorar la tecnología productiva ancestral que está en armonía con la madre tierra para poder coadyuvar al desarrollo regional, sin dejar de lado los avances científicos y tecnológicos que tienen valor en nuestro contexto.

TICs en el aprendizaje: Las Tecnologías de Información y Comunicación (TIC) se han convertido en una poderosa herramienta didáctica que promueven la colaboración entre los alumnos, centrarse en sus aprendizajes y mejoran la motivación y el interés por el estudio. Asimismo, generan la integración y estimulan el desarrollo de ciertas habilidades intelectuales tales como el razonamiento la resolución de problemas, la creatividad y la capacidad de aprender a aprender.

Integración: Para Estermann (2015), el individuo, visto exclusivamente a través de su individualidad, sería entonces concebido como incompleto; solo existe porque se complementa con los demás, incluso con los opuestos. Entonces el hombre con pensamiento indígena andino no excluye las fuerzas opuestas, sino que encuentra una correlación entre estas para afirmar una organización integral, por lo tanto, superior.

Creatividad e innovación: La creatividad es la clave del éxito y la innovación es el instrumento para plantear nuevas formas de trabajo para mejorar y lograr el desarrollo personal y colectivo; por ello, se debe educar para generar nuevos conocimientos en todos los campos del saber, mediante el desarrollo de diversas investigaciones e indagaciones para crear e innovar en bien de la sociedad según el avance científico y tecnológico. 
Congreso Internacional de Gestión Educativa, 06 y 07 de noviembre de 2020.

Pontificia Universidad Católica del Perú

Calidad: La calidad en la educación es una aspiración constante de todos los sistemas educativos e implica un juicio de valor respecto del tipo de educación que se brinda con la finalidad de formar un ideal de persona y de sociedad que están condicionados por factores ideológicos, culturales y políticos en un momento dado. Estos factores son dinámicos y cambiantes porque la definición de una educación de calidad varía según los períodos. Principios de la cosmovisión andina Quinatoa (2014), refiere que en la naturaleza, los seres humanos y la Pachamama (Madre Tierra) son un todo que viven relacionados perpetuamente.

\section{La propuesta}

La propuesta de una educación a distancia e interrupción de los servicios presenciales, generan nuevas necesidades en la prestación del servicio educativo, lo que hizo que se propongan y desarrollen acciones desde un gobierno digital, implementado ya antes de la pandemia y declaratoria de emergencia sanitaria a fin de garantizar la modernización de la gestión educativa e ir consolidando una gestión escolar pública asentado en el escritorio limpio, implementándose una plataforma virtual exclusiva para facilitar los flujos de tramitación institucional, al que se denomino Sistema Integrado para Instituciones Educativas (SIIE).

Implementar este gobierno digital, necesariamente debería estar sustentado en base a la práctica comprometida y consciente de los diferentes actores de la comunidad educativa de los principios de la racionalidad andina: la relación, la correspondencia, la complementariedad y la reciprocidad que se convierten en un soporte intercultural básico para mejorar la calidad de la educación.

La metodología de uso se basa en principios y normas de la racionalidad andina:

Allin Kawsay / Wali Jakaña / Vivir bien

Allin Munay / Wali Munaña/ Querer bien

Allin Yachay / Wali Yatiqaña / Aprender bien

Allin Ruray / Wali Luraña / Hacer bien

En medio de esta práctica de la gestión educativa moderna e intercultural, resulta también una necesidad el fortalecer y consolidar la autonomía de las instituciones educativas para la toma de decisiones y ejecución del servicio educativo propiamente dicho, orientado a fortalecer la mejora de la calidad de los aprendizajes. El artículo 66 de la Ley General de Educación 28044 establece a la Institución Educativa “...como comunidad de aprendizaje, es la primera y principal instancia de gestión del sistema educativo descentralizado. En ella tiene lugar la prestación del servicio, puede ser público o privada."

Las acciones implementadas han permitido asumir el servicio educativo con pertinencia y oportunidad. La estrategia "Aprendo en Casa" fue asumido por docentes, estudiantes y padres de familia para el cumplimiento de los procesos de Gestión que realizan los directores de las Instituciones Educativas, en términos de sostenibilidad de los resultados, mayor comunicación y el "buen vivir" como soporte. 
Congreso Internacional de Gestión Educativa, 06 y 07 de noviembre de 2020.

Pontificia Universidad Católica del Perú

Desarrollo de la experiencia: El Ministerio de Educación desde el año 2007 inició un proceso de modernización de la gestión pública de la educación basado en "presupuestos por resultados" y a partir del año 2014 se establecen compromisos de desempeño para el cumplimiento de la UGEL a fin de obtener asignaciones económicas como incentivos, por lo que desde el inicio de la actual gestión de la UGEL Puno se fueron implementando estrategias a partir de las premisas: "Innovando liderazgo y gestión" y "Consolidando la autonomía de las instituciones educativas". Una de las actividades más importantes fue la ejecución de las reuniones ordinarias de carácter mensual con directivos de las instituciones educativas cuya naturaleza de participación era voluntaria y más bien motivada por la práctica de los principios de la racionalidad andina y el interés generado por una hoja de ruta propuesta con anticipación que siempre tiene un componente de asistencia y soporte técnico normativo, así como niveles de coordinación institucional de la política educativa, nacional, regional y local, de estas reuniones y atendiendo necesidades de modernizar, agilizar y simplificar la gestión surge la iniciativa de la creación de implementar la plataforma virtual a partir del año 2019.

El uso de la plataforma virtual le da sostenibilidad y mejora a la calidad de los aprendizajes de los estudiantes, para tal fin se realizaron las adaptaciones al trabajo remoto y/o teletrabajo. Las reuniones mensuales se reinician después de tres meses de suspensión por el aislamiento social, asumiendo que éstas fueran de manera virtual, por lo tanto se implementó una sala de conferencias utilizando la plataforma virtual zoom y el fortalecimiento del Sistema Integrado para Instituciones Educativas (SIIE) que está siendo utilizado cada vez más con mayor frecuencia. Esto ha permitido una mayor comunicación y coordinación en la gestión de las instituciones educativas, que tuvieron como complemento y necesidad fortalecer las capacidades digitales tanto de los trabajadores de la sede administrativa para la atención al público usuario como también de los directivos y docentes de las instituciones educativas en el trabajo de acompañar los aprendizajes, así se iniciaron las capacitaciones virtuales con una serie de webinar y cursos virtuales en línea sobre el uso de herramientas digitales que posibiliten afrontar la gestión y hacer que el gobierno digital sea eficiente. Así también las exigencias de la educación a distancia a través de la estrategia "aprendo en casa", requieren, como complemento, el soporte emocional brindado a través de espacios radiales y televisivos propios de la UGEL que son retransmitidos en las redes sociales.

Lecciones aprendidas: El desarrollo de la experiencia nos ha permitido comprender que podemos adaptarnos a las necesidades de una nueva normalidad de la gestión educativa utilizando las tecnologías de comunicación y aprendizaje para garantizar la continuidad del servicio educativo en términos de gestión y el desarrollo de la enseñanza aprendizaje de los estudiantes como también docentes, sabíamos que los hogares se habían convertido en salones de clase y centros de trabajo sin descuidar las interrelaciones de familia que al mismo tiempo se convirtieron en maestros sustitutos, sin embargo se complementaban con el soporte de acompañamiento basado en la telefonía digital que tendría que ofrecía el docente de aula y/o área que como ya sabíamos en alto porcentaje no estaban preparados para enfrentar estos retos respecto a los entornos virtuales y tecnológicos, lo cual fue conscientemente solicitado en términos de capacitación por los directores cuando participaban en las reuniones mensuales y también cuando hacían uso de la plataforma virtual del Sistema Integrado para 
Congreso Internacional de Gestión Educativa, 06 y 07 de noviembre de 2020.

Pontificia Universidad Católica del Perú

Instituciones Educativas, que permitió, entre otras acciones: El desarrollo de Talleres Virtuales gratuitos denominado Tecno TIC Puno dirigido a docentes para el manejo de entornos y herramientas virtuales. Difusión permanente para que los directivos, docentes y PP.FF. conozcan y promuevan participación en la estrategia "Aprendo en casa". Coordinación con autoridades locales: Municipalidad y otros para garantizar la prestación de la educación a distancia. La elaboración y actualización del directorio del personal de las II.EE. Desarrollo de capacitación virtual en manejo de herramientas virtuales para trabajadores de la UGEL Puno, directores, subdirectores y docentes, implementación del Sistema de Trámite Documentario Virtual. Presentación virtual de documentación por los directivos.

Son también lecciones aprendidas el manejo de información sobre las condiciones de conectividad y acceso a medios de comunicación de las II.EE. Apoyo para el cuidado y vigilancia de las IIEE, en materia de seguridad y salud del personal, contra el COVID19. Organización de especialistas para realizar asistencias técnicas pedagógicas por niveles, áreas y modalidades educativos. Seguimiento y apoyo a los directores utilizando medios virtuales de comunicación y la plataforma SIMON. Asistencia técnica a los directivos según los resultados del monitoreo y nudos críticos identificados. Generación y/o fortalecimiento de redes de soporte entre Directivos y/o docentes por niveles, con refuerzo en el liderazgo y la autonomía de las I.E. Difusión de las buenas prácticas de educación a distancia de los docentes o directivos de II.EE. haciendo uso de entornos virtuales en el contexto de la emergencia sanitaria, como acción complementaria a la estrategia "Aprendo en Casa".

\section{Agradecimientos}

Al equipo de especialistas del área de Gestión Pedagógica, a los Directores, de instituciones educativas, a los padres de familia y a las autoridades locales de la provincia.

\section{Referencias bibliográficas}

Ministerio de Educación del Perú (2019) Diseño Curricular Nacional.

Ministerio de Educación del Perú (2020) R.M. N 160-2020-MINEDU

Dirección Regional de Educación de Puno (2019) Proyecto Educativo Regional Puno.

Unidad de Gestión Educativa Local de Puno (2020) Proyecto Educativo Local.

Ávila, J. (2014). El enfoque del buen vivir como una visión colectiva. Revista de Ciencias Sociales Vol. 9, $\mathrm{N}^{\circ} 1$.

Bustamante, P. (2015). Objetivos de Desarrollo Sostenible e Inclusión Social. Ministerio de Educación. Lima. Perú.

Grupo Propuesta (2016). Gestión estratégica de la educación en Puno. El currículo y la identidad como ejes del cambio. Lima Perú.

Treviño, E. Villalobos, C. y Baeza, A. (2016). Recomendaciones de Políticas Educativas en América Latina en base al TERCE. UNESCO. Santiago de Chile. 
Congreso Internacional de Gestión Educativa, 06 y 07 de noviembre de 2020.

Pontificia Universidad Católica del Perú

UNESCO (2016). Tecnologías digitales al servicio de la calidad educativa. Una propuesta de cambio centrada en el aprendizaje para todos. Santiago de Chile.

UNICEF. (2017). Políticas educativas para transformar la educación secundaria. Fondo de las Naciones Unidas para la Infancia (UNICEF), Flacso. Argentina. 